\title{
1 Complex feline disease mapping using a dense genotyping array
}

2 Isabel Hernandez ${ }^{1 \uparrow}$, Jessica J. Hayward ${ }^{2 \pi *}$, Jeff A. Brockman ${ }^{3}$, Michelle E. White ${ }^{4}$, Lara

3 Mouttham $^{5}$, Elizabeth A. Wilcox ${ }^{5}$, Susan Garrison ${ }^{5}$, Marta G. Castelhano ${ }^{5}$, John P. Loftus ${ }^{1}$, Filipe

4 Espinheira Gomes ${ }^{1}$, Cheryl Balkman ${ }^{1}$, Marjory B. Brooks ${ }^{6}$, Nadine Fiani ${ }^{1}$, Marnin Forman ${ }^{7}$, Tom

5 Kern $^{1}$, Bruce Kornreich ${ }^{1}$, Eric Ledbetter ${ }^{1}$, Santiago Peralta ${ }^{1}$, Angela M. Struble ${ }^{1}$, Lisa Caligiuri ${ }^{1}$,

6 Elizabeth Corey ${ }^{5}$, Lin Lin ${ }^{5}$, Julie Jordan ${ }^{5}$, Danny Sack ${ }^{1}$, Adam R. Boyko ${ }^{2}$, Leslie A. Lyons ${ }^{8}$, Rory

$7 \quad$ J. Todhunter ${ }^{1}$

$9{ }^{1}$ Department of Clinical Sciences, College of Veterinary Medicine, Cornell University, Ithaca, 10 New York, United States of America

${ }^{2}$ Department of Biomedical Sciences, College of Veterinary Medicine, Cornell University, Ithaca, New York, United States of America

${ }^{3}$ Pet Nutrition Center, Hill's Pet Nutrition, Topeka, Kansas, United States of America

${ }^{4}$ Bioinformatics and Integrative Biology, University of Massachusetts Medical School,

Institute of MIT and Harvard, Cambridge, Massachusetts, United States of America

${ }^{5}$ Cornell Veterinary Biobank, College of Veterinary Medicine, Cornell University, Ithaca, New York, United States of America

${ }^{6}$ Department of Population Medicine and Diagnostic Services, College of Veterinary Medicine,

${ }^{7}$ Cornell University Veterinary Specialists, Stamford, Connecticut, United States of America

${ }^{8}$ Department of Veterinary Medicine \& Surgery, College of Veterinary Medicine, University of

*Corresponding author:

33 Email: jessica.hayward@cornell.edu 
35 These authors contributed equally to this work.

36

37 Short title: Feline complex disease mapping

38

39 Keywords: feline complex disease, genome-wide association study, biobank

40 


\section{Abstract}

43 utility within breeds, and its use has led to the identification of variants associated with Mendelian

44 traits in purebred cats. However, compared to single gene disorders, association studies of complex

45 diseases, especially with the inclusion of random bred cats with relatively low linkage

46 disequilibrium, require a denser genotyping array and an increased sample size to provide

47 statistically significant associations. Here, we undertook a multi-breed study of 1,122 cats, most

48 of which were admitted and phenotyped for nine common complex feline diseases at the Cornell

49 University Hospital for Animals. Using a proprietary 340k single nucleotide polymorphism

50 mapping array, we identified significant genome-wide associations with hyperthyroidism, diabetes

51 mellitus, and eosinophilic keratoconjunctivitis. These results provide genomic locations for variant

52 discovery and candidate gene screening for these important complex feline diseases, which are

53 relevant not only to feline health, but also to the development of disease models for comparative

54 studies.

\section{Introduction}

There are 365 hereditary disorders of cats listed on OMIA (Online Mendelian Inheritance

58 in Animals, https://omia.org/home/ accessed June 7th, 2021), of which only 119 (32.6\%) are

59 Mendelian traits and only $136(37.3 \%)$ have likely causal variants. Clearly, there are a large

60 number of feline diseases whose genetic basis is still unknown. Moreover, 230 of these hereditary

61 feline disorders are potentially good models for human disease. 
63 Random bred cats are the most common cats in American households, accounting for $84 \%$ of the

64 population in the United States [1]. Random bred cats comprised 89\% of cats admitted the Cornell

65 University Hospital for Animals (CUHA) in the last 15 years, thus providing an important

66 spontaneous source of DNA for increasing sample sizes of genetic mapping studies.

68 Compared to purebreds, random bred cats have shorter linkage disequilibrium, due to the large

69 number of generations since the origin of the random bred cat population, with archaeological

70 evidence of a human and cat burial site as old as 9,500 years [2]. The genetic heterogeneity of

71 random bred cats, the additive effect of many genes, and their environmental interaction makes

72 discovering variants contributing to complex diseases more challenging than for Mendelian traits

73 [3]. At least a few Mendelian traits have been mapped in random bred cats, including spongy

74 encephalopathy, Glanzmann thrombasthenia, and inflammatory linear verrucous epidermal nevus

75 [4-6]. Additional factors that make the discovery of complex disease genetic mechanisms difficult

76 include sample size, phenotyping accuracy, mapping array marker density, and access to whole

77 genome sequences for variant discovery [7].

79 The current 63k Illumina feline single nucleotide polymorphism (SNP) mapping array has been

80 used successfully within breeds to map variants for Mendelian diseases. Examples include the

81 discovery of the WNK4 variant that causes hypokalemia in Burmese cats [8], a region on

82 chromosome E1 associated with progressive retinal atrophy in Persian cats [9], a causal variant in

$83 C O L Q$ for hereditary myopathy in Devon Rex and Sphynx cats [10], refinement of the region on

84 chromosome B4 associated with craniofacial structure and frontonasal dysplasia in Burmese cats

85 [11], a region on chromosome A3 associated with an inherited neurologic syndrome in a family of 
86 Oriental cats [12], and a dominant channelopathy variant causing osteochondrodysplasia in

87 Scottish Fold cats [13]. This array has also been used in a limited number of within-breed genome

88 wide association studies (GWAS) for complex disease [14,15], but there are no reports of GWAS

89 performed with an across-breed design.

91 Here, we genotyped 1,122 cats using a one-time proprietary Illumina high density 340k SNP

92 mapping array designed by Hill's Pet Nutrition, in an effort to identify genetic underpinnings for

93 nine complex diseases. Our samples consisted of a mix of 31 purebreds and 905 random bred cats,

94 the majority of which were domestic shorthairs. This array improves upon the density of the current

95 commercial $63 \mathrm{k}$ array by a factor of $>5$. As quality control and to validate the accuracy of the $340 \mathrm{k}$

96 array, we performed a GWAS for the Orange coat color locus and for Factor XII deficiency, which

97 are known to be associated with a region on chromosomes $X$ and A1, respectively [16-19].

99 The complex diseases included in this study were hypertrophic cardiomyopathy (HCM),

100 hyperthyroidism, diabetes mellitus (DM), chronic kidney disease (CKD), chronic enteropathy,

101 inflammatory bowel disease (IBD), small cell alimentary lymphoma (SCAL), hypercalcemia, and

102 feline eosinophilic keratoconjunctivitis (FEK). These diseases are among the most common

103 complex diseases of cats admitted to CUHA and are some of the most common and important

104 feline diseases in clinical veterinary practice [20].

105

106 We used both a linear mixed model (LMM) and a multi-locus method called Fixed and random

107 model Circulating Probability Unification (FarmCPU) to perform GWAS, and together identified

108 loci significantly associated with hyperthyroidism, DM, FEK, and IBD. Additionally, we 
109 identified suggestive loci for the diseases HCM and hypercalcemia. Here, we describe the largest

110 genetic mapping study of feline complex diseases with the densest mapping array ever performed.

112 Results

\section{Validation of array}

114 Principal component analysis (PCA) was performed using all genotyped cats that passed

115 quality control, and showed that there was no batch effect due to the 11 sequential plates used for 116 genotyping (Fig. 1A). The first two components, principal component (PC)1 and PC2, explained

$11731.3 \%$ of the total genetic variation. The cluster that separates on PC2 in this PCA includes 40 cats

118 from a closed colony of domestic shorthair (DSH) cats from a local breeding facility, genotyped

119 mainly on plates 7 and 11. Principal component analysis of the genotypes of all 221 purebred cats

120 showed that PC1 separates western breeds, like Manx and Persian, from eastern breeds, like

121 Tonkinese and Burmese (Fig. 1B). This eastern-western distribution of breeds is also seen on PC1

122 of the PCA of all cats (Fig. 1A) and has been shown previously using the 63k genotyping array

$123[16,21-23]$. PC2 of the purebred cat PCA separates the Devon Rex cats from the other breeds. The

124 first two components of the purebred cat PCA explained only $16.4 \%$ of the total genetic variation,

125 much less than the $38.4 \%$ explained by the first two components of a PCA using the $63 \mathrm{k}$ array $126[23]$.

128 Figure 1. Principal Component Analysis of cat genetic structure. Dimensions PC1 and PC2

129 are shown. (A) All 1,122 cats that passed QC, color-coded by genotyping plate (1 to 11), showing 130 the absence of a batch effect. PC1 shows the eastern-western breed distribution. The cluster of cats 131 that separate on PC2 is from a local colony that were genotyped on plates 2 (dark blue), 7 (brown), 
132 and 11 (orange). (B) 221 purebred cats color-coded by breed, showing the eastern-western breed

133 distribution on PC1. The Devon Rex breed (dark green) separates on PC2.

\section{GWAS positive controls}

As a positive control, we performed a GWAS on the presence of orange fur in random bred 137 cats (90 orange fur, 121 black/brown fur). Using the linear mixed model in GEMMA, we identified 13825 significant associations on a region of chromosome $X$ between 102,884,842 bp and 112,136,902 139 bp (Fig. 2A; S1 Table). The most significantly associated SNP in both the LMM and FarmCPU 140 GWAS is at $110,230,748 \mathrm{bp}\left(P=1.8 \times 10^{-102}\right.$ and $P=2.2 \times 10^{-97}$, respectively $)$, located within an intron 141 in the gene Ecto-NOX Disulfide-Thiol Exchanger 2 (ENOX2). This region is known to contain the

142 Orange cat coloration locus [16-18] and the most significant SNP is within the 1.5 Mb haplotype 143 block identified by Gandolfi et al. (2018). A linkage disequilibrium (LD) plot of this region 144 showed that the 340k array has very few markers between $105-110 \mathrm{Mb}$ on chromosome $\mathrm{X}$, and 145 only 4 markers within the $1.5 \mathrm{Mb}$ haplotype block remain after minor allele frequency (MAF) and 146 missingness filters, preventing the refinement of this region (S1A Fig.).

148 Figure 2. Manhattan and quantile-quantile (QQ) plots for GWAS positive controls. X axis 149 represents the chromosomal SNP position and Y axis represents the $-\log _{10}(P$-value $)$. The QQ plots 150 show observed versus expected $P$-values for each SNP. (A) Orange coat color locus, showing the 151 significant association on chromosome $\mathrm{X}\left(P=1.8 \times 10^{-102}\right)$. (B) Factor XII deficiency, showing the 152 significant associations on chromosomes A1 and C2. The red line on the Manhattan plots shows 153 the Bonferroni-corrected significance threshold and the blue line on the Manhattan plot in B shows 
154 the Bonferroni-corrected significance threshold calculated using unlinked SNPs. The genomic 155 inflation factor $(\boldsymbol{\lambda})$ is shown on each QQ plot.

157 As a second positive disease control, we performed a GWAS for factor XII deficiency, using 19 158 affecteds and 34 controls. The LMM in GEMMA identified four significant associations on 159 chromosome A1, between 175,333,103 bp and 175,445,463 bp, which reside within $63 \mathrm{~kb}$ of the 160 gene Coagulation Factor XII (F12) (Fig. 2B, S1B Fig.). The most significant association using the

161 FarmCPU method was the same A1 association at $175,445,463$ bp $(P=1.4 \times 10-19)$. Two high162 frequency variants in the gene $F 12$ have previously been reported in cats with factor XII deficiency 163 [19]. Other significant associations were also identified in the factor XII GWAS by both models, 164 on chromosomes C2, C1, D2, F1 and D3.

\section{Disease GWAS}

Across-breed case/control GWAS was conducted for the diseases HCM, hyperthyroidism,

168 DM, CKD, chronic enteropathy, IBD, SCAL, FEK, hypercalcemia, and all gastrointestinal

169 phenotypes (chronic enteropathy, IBD, and SCAL) merged together. Significance thresholds were

170 calculated using the Bonferroni correction on all SNPs included in each GWAS, while suggestive

171 thresholds were calculated using the Bonferroni correction on a pruned set of unlinked SNPs.

173 Three significant and two suggestive associations were identified above the genome-wide 174 thresholds by the LMM GWAS in GEMMA (Table 1). The FarmCPU GWAS showed very similar 175 results to the LMM GWAS, with significant associations for DM and hyperthyroidism (Table 1; 176 S2 Table). However, the FEK association was not significant in the FarmCPU GWAS while the 
177 IBD association was significant (Table 1; S2 Table). Since the results from the two methods were

178 so similar, we have chosen to focus illustrating the results of the LMM GWAS. Genomic inflation

179 factors, $\square$, are all $<1.07$ (range of 0.997-1.052, average 1.016 for LMM; range of 1.013-1.062,

180 average 1.033 for FarmCPU), showing successful control for underlying population structure.

182 Table 1: Significant and suggestive associations identified for complex diseases using an 183 across-breed GWAS design. Results are shown for both the LMM and FarmCPU GWAS.

\begin{tabular}{|c|c|c|c|c|c|}
\hline $\begin{array}{l}\text { Disease } \\
\text { (number cases, } \\
\text { number controls) }\end{array}$ & $\begin{array}{l}\text { GWAS } \\
\text { model }\end{array}$ & Chr: bp & $P$-value & $\begin{array}{l}\text { Allele frequency } \\
\text { (cases, controls) }\end{array}$ & Candidate genes \\
\hline $\begin{array}{l}\text { Hyperthyroidism } \\
(310,134)\end{array}$ & $\begin{array}{c}\text { LMM } \\
\text { FarmCPU }\end{array}$ & $\begin{array}{l}\text { B2: } 121,565,607 \\
\text { B2: } 121,565,607\end{array}$ & $\begin{array}{l}1.25 \times 10^{-7} \\
1.36 \times 10^{-7}\end{array}$ & $0.037,0.127$ & $A R G 1, M E D 23$ \\
\hline $\begin{array}{l}\text { DM } \\
(67,366)\end{array}$ & $\begin{array}{c}\text { LMM } \\
\text { FarmCPU }\end{array}$ & $\begin{array}{l}\text { D4: } 83,583,678 \\
\text { D4: } 83,583,678\end{array}$ & $\begin{array}{l}1.62 \times 10^{-7} \\
2.55 \times 10^{-7}\end{array}$ & $0.366,0.172$ & $\begin{array}{c}\text { olfactory receptors, } \\
\text { PTGS1 }\end{array}$ \\
\hline $\begin{array}{l}\text { FEK } \\
(15,40)\end{array}$ & $\begin{array}{c}\text { LMM } \\
\text { FarmCPU }\end{array}$ & $\begin{array}{l}\text { E3: } 34,663,327 \\
\text { E3: } 34,663,327\end{array}$ & $\begin{array}{l}1.62 \times 10^{-7} \\
1.79 \times 10^{-6} \#\end{array}$ & $0.100,0.638$ & TNFRSF17 \\
\hline $\begin{array}{l}\text { IBD } \\
(47,33)\end{array}$ & $\begin{array}{c}\text { LMM } \\
\text { FarmCPU }\end{array}$ & $\begin{array}{l}\text { B4: } 10,941,073 \\
\text { B4: } 10,941,073\end{array}$ & $\begin{array}{l}2.75 \times 10^{-6} \# \\
9.54 \times 10^{-8}\end{array}$ & $0.216,0.533$ & N/A \\
\hline $\begin{array}{l}\text { HCM } \\
(85,53)\end{array}$ & $\begin{array}{c}\text { LMM } \\
\text { FarmCPU }\end{array}$ & $\begin{array}{l}\text { E3: } 3,583,882 \\
\text { E3: } 3,583,882\end{array}$ & $\begin{array}{l}2.76 \times 10^{-7 \wedge} \\
1.00 \times 10^{-6} \#\end{array}$ & $0.295,0.604$ & $S D K 1$ \\
\hline Hypercalcemia & LMM & $\mathrm{C} 1: 19,508,050$ & $6.81 \times 10^{-7 \wedge}$ & $0.300,0.085$ & N/A \\
\hline
\end{tabular}




$(25,443) \quad$ FarmCPU $\quad \mathrm{C} 1: 19,508,050 \quad 3.05 \times 10^{-7 \wedge}$

$\wedge$ suggestive association based on unlinked SNPs

185 \# not significant

Hyperthyroidism. For hyperthyroidism, we found a solitary significant association on

Figure 3. Manhattan, quantile-quantile (QQ), and LD plots for case-control disease association on chr E3. On Manhattan plots, the red line is Bonferroni-corrected significance unlinked SNPs. Inflation factors $(\boldsymbol{\lambda})$ are shown on QQ plots. On LD plots, the colors indicate the amount of LD $\left(\mathrm{r}^{2}\right)$ with the most significant SNP, ranging from black $\left(\mathrm{r}^{2}<0.2\right)$ to red $\left(\mathrm{r}^{2}>0.8\right)$. 
206 (cyclooxygenase-1), is located within $123 \mathrm{~kb}$ downstream of, although not in LD with, our 207 significant association. This locus on D4 affects the risk of DM in DSH and Maine Coon cats, but 208 not in cats of other breeds and DLH cats (S3 Table).

210 Feline eosinophilic keratoconjunctivitis. We identified a significant association for FEK

$211\left(P=1.62 \times 10^{-7}\right)$ in the LMM GWAS, with a marker on chromosome E3, located $10.5 \mathrm{~kb}$ from the 212 gene TNFRSF17 (tumor necrosis factor receptor superfamily, member 17) (Fig. 3C). The second 213 most significant association with this disease did not reach significance $\left(P=3.1 \times 10^{-6}\right)$ but is located 214 within the gene TNFRSF21 (tumor necrosis factor superfamily, member 21). Both TNFRSF17 and 215 TNFRSF21 belong to the tumor necrosis factor receptor superfamily, and TNFRSF21 is expressed 216 in the eye [24]. The E3 locus affects the risk for FEK in DSH cats (S3 Table).

218 IBD. A significant association $\left(P=9.54 \times 10^{-8}\right)$ for IBD was identified using the FarmCPU GWAS.

219 The marker is on chromosome B4 near the genes ECHDC3 (enoyl-CoA hydratase domain 220 containing 3 ) and USP6NL (ubiquitin-specific protease 6 N-terminal like) (S2 Fig.). ECHDC3 has

221 a role in fatty acid biosynthesis and has been found to have an increased expression in the brains 222 of Alzheimer's patients [25] while USP6NL is a GTPase-activating protein for Rabs and is up223 regulated in several cancers, including breast and colorectal cancers [26,27]. The B4 significant 224 locus affects risk for IBD in DSH and DLH cats (S3 Table).

226 HCM. The LMM GWAS for HCM reached suggestive significance $\left(P=2.76 \times 10^{-7}\right)$ with a marker 227 on chromosome E3, located within the gene SDK1 (sidekick cell adhesion molecule 1) (Fig. 4A), 
which is expressed especially in the kidney and retina [28,29] but has also been associated with hypertension [30]. This suggestive E3 locus affects risk for HCM in DSH cats (S3 Table).

231 Figure 4. Manhattan, quantile-quantile (QQ), and LD plots for case-control disease

233 SNP position and $\mathrm{Y}$ axis represents the $-\log _{10}(P$-value $)$. The QQ plots show observed versus $\left(\mathrm{r}^{2}<0.2\right)$ to red $\left(\mathrm{r}^{2}>0.8\right)$.

Hypercalcemia. The hypercalcemia GWAS produced a suggestive association $\left(P=6.81 \times 10^{-7}\right.$ in

244 The enzyme encoded by PAFAH2 acts to protect the cell from oxidative cytotoxicity [31], while

245 the protein encoded by STMN1 is involved in regulating the microtubule cytoskeleton, including mitotic spindle formation [32]. The C1 locus affects risk for hypercalcemia in DSH cats (S3 Table).

248 Genome-wide association studies of the other complex diseases, CKD, SCAL, chronic 249 enteropathy, and merged GI phenotypes did not produce a significant or suggestive association 250 using either the LMM or FarmCPU GWAS (S3 Fig., S2 Table, S4 Table). 


\section{Discussion}

In this study, we identified significant associations for common, clinically relevant,

254 complex diseases in a population of 1,122 random and purebred cats, using a dense genotyping

255 array. While a similar study was previously performed in dogs [33], this is the largest GWAS

256 disease study in cats reported to date, conducted in a heterogeneous natural population including

$25780 \%$ random bred cats. Further advantages of the current study design were the careful

258 phenotyping of aged control cats, accurate phenotyping of diseased participants by specialists

259 performed in an academic clinical setting, and a mapping array approximately 5-fold denser than

260 the current 63k array. Additionally, the quality of the biospecimens used and its associated data

261 demonstrate the importance of using an accredited resource such as the Cornell Veterinary

262 Biobank.

263

264 As a positive control, we identified significant associations for the Orange coat color locus and

265 factor XII deficiency at the F12 gene locus. Although the F12 locus was the most significant

266 association using both LMM and FarmCPU models, three and five other significant SNPs were

267 identified in the factor XII deficiency GWAS, respectively. A BLAT [34] search showed that the

268 flanking region of the three SNP maps to many places in the feline genome, including chromosome

$269 \mathrm{~A} 1: 175 \mathrm{Mb}$, the location of the gene F12. Thus, it appears that there may be some non-specific

270 binding with the A1 probe. However, factor XII deficiency is affected by several different loci

271 across the genome, as shown in humans [35].

272 
273 The majority of the cats included in our analyses are random bred cats, which generally have

274 shorter LD than purebreds [3], because they have not been subject to selective breeding for specific

275 traits. Further, we are mapping complex diseases, which usually consist of many variants each

276 contributing a small effect, and have not been subjected to artificial selection, resulting in shorter

277 LD surrounding the causal variant. As a result of investigating complex diseases in a

278 predominantly random bred cat population, we do not expect to see the stacking of SNPs that are

279 seen in GWAS studies of morphological traits, especially in purebred cats.

280

281 Using a case/control approach, we performed GWAS with both a LMM and FarmCPU, and found

282 very similar results. Both methods identified significant associations for hyperthyroidism and DM,

283 and the FEK association was significant in the LMM GWAS while the IBD association was

284 significant in the FarmCPU GWAS. Furthermore, the same SNPs were identified as the most

285 significant associations by both models.

287 For hyperthyroidism, the candidate gene $A R G 1$ encodes Arginase 1, a cytosolic enzyme that 288 participates in the urea cycle and is expressed in the liver [36]. Another nearby candidate gene,

289 MED23, encodes a component of the thyroid hormone receptor (TR) associated protein complex.

290 As such, it interacts with, and facilitates, TR function. Variants in the TR have been associated

291 with thyroid hormone resistance, for which the clinical presentation is very similar to

292 thyrotoxicosis [37]. This is the first GWAS for feline hyperthyroidism reported and our finding 293 represents a novel locus. Somatic variants in the thyroid-stimulating hormone receptor (TSHR)

294 gene have been previously reported, but those variants were identified in DNA extracted from the

295 affected thyroid glands of hyperthyroid cats [38]. 
297 The significant DM locus includes many olfactory receptor genes. Genetic and epigenetic

298 variation, and the resulting functional changes, in olfactory receptors have been associated with

299 taste, food intake, and satiety [39,40]. These differences may contribute to obesity risk and risk of

300 DM. Mouse olfactory receptor gene OLFR15 has been shown to be expressed in pancreatic beta-

301 cells and to regulate the secretion of insulin [41]. The other interesting gene near our significant

302 D4 association, although not quite within the LD region of interest, PTGS1, encodes an enzyme

303 that converts arachinodate into prostaglandin, which is involved in glucose homeostasis [42]. This

304 gene has been associated with human DM [43,44]. Our findings constitute a novel locus associated

305 with DM. Previous studies have identified several loci associated with DM in Australian Burmese

306 cats $[15,45]$ and a polymorphism in melanocortin receptor 4 (MCR4) associated with DM in obese

307 domestic shorthair cats [46].

309 For eosinophilic keratoconjunctivitis, we identified a significant association in the LMM GWAS

310 near the gene TNFRSF17. This is especially promising, and warrants further investigation because

311 of its role in the innate and adaptive immune response. In patients with allergic asthma, eosinophils

312 infiltrate the bronchial wall and lumen, and the bronchial epithelium is often damaged [47]. These

313 pathological findings are associated with aberrant $\mathrm{T}$ helper 2 (Th2) cell-mediated immune

314 responses. Interleukin-5, which is produced by Th2 cells, and the chemokine eotaxin are key

315 players for the proliferation, differentiation, activation and mobilization of eosinophils $[48,49]$. In

316 knockout mice studies, NF-kappa-B, a transcription factor that is activated by the TNFRSF17 and

317 TNFRSF21 genes, was found to play an important role in Th2 cell differentiation and is therefore

318 required for induction of allergic airway inflammation $[48,50]$. Similar to knockout mice with 
319 allergic asthma, it is possible that animals affected with FEK have an abnormal NF-kappa-B

320 activation due to defective expression of TNFRSF17 and TNFRSF21 genes, as suggested by the

321 current GWAS study.

322

323 The IBD significant association, as identified by the FarmCPU method only, was located near the

324 genes ECHDC3 and USP6NL. Neither of these genes are good candidates for a gastroenteropathy

325 phenotype.

327 The first of the two suggestive associations, HCM, was located in the gene SDK1. A polymorphism

328 in SDK1 was found to be associated with hypertension in a study of over 5,000 Japanese

329 individuals [30] but the function of this gene related to hypertension has not been described.

330 Finally, the LD region surrounding the suggestive association for hypercalcemia contained the

331 genes $P A F A H 2$ and STMN1, neither of which have been associated with hypercalcemia previously.

333 Despite the use of a dense genotyping array, across-breed GWAS for CKD, SCAL, chronic

334 enteropathy, and merged gastrointestinal phenotypes did not reach statistical genome-wide

335 significance using either single-locus or multi-locus models. We believe that larger cohorts may

336 be needed due to the genetic architecture of these diseases, especially chronic enteropathy for

337 which we had fewer than 50 cases in the respective GWAS.

339 By not restricting our analyses to a single breed, we were able to include a relatively large sample

340 size for some of our phenotypes, thereby increasing statistical power to identify significant loci. In 
341 a study of this kind, especially if the majority of cats are randomly bred, LD is shorter, resulting

342 in smaller regions of interest and narrowing the list of potential candidate genes.

344 Nevertheless, for some other phenotypes, we had an unbalanced proportion of cases and controls.

345 This is due to the fact that accumulation of samples takes a long time, in part because donating

346 samples is an opt-in process in our hospital, and because of the difficulty of recruiting universal

347 controls. It is an unanswered question how many samples are required for a robust across-breed,

348 complex disease GWAS study in cats, but canine simulation studies indicate that 500-1000 cases

349 and controls, plus a further increase in array marker density, would substantially increase loci

350 discovery in dogs [33].

352 Follow-up analyses using an independent cohort of phenotyped cats are needed to validate the

353 associations we identified in this group of genotyped cats. Further studies involving investigation

354 of the regions surrounding the significant associations are needed to determine causal variants for

355 these complex diseases. Use of the $>300$ whole genome sequences provided by the 99Lives Feline

356 Genome Consortium will allow variant discovery within candidate genes in the intervals of

357 interest. Finally, functional studies will be required to confirm causal variants.

359 In this research, we used an across-breed GWAS design with a $~ 5$-fold denser genotyping array 360 than currently available, to identify significant associations with important common feline 361 complex diseases. We demonstrated that a well-curated, hospital-sourced population can be used

362 effectively for mapping studies. We also demonstrated the benefit of such a dense mapping array, 363 propelling the field of complex feline disease genetics forward. Further, these results can be used 
364 to develop new diagnostic tests to assist veterinarians in identifying diseases earlier and allowing

365 the implementation of early preventative measures. Breeders could improve their practices by

366 identifying cats with optimum genetic value and owners could make informed decisions regarding

367 the health of cats. This is particularly important in this era of personalized medicine. The shared

368 environment of cats and their owners further enhances the value of domestic cats as models of

369 lifestyle disease common to both species.

\section{Materials and methods}

\section{Banking biospecimens and associated data}

The 1,122 feline biospecimens used for this project were selected from the Cornell

374 Veterinary Biobank (CVB), a core resource at the Cornell University College of Veterinary

375 Medicine, which has been collecting and processing whole blood samples from feline patients

376 admitted to the Cornell University Hospital for Animals (CUHA) since 2006. Biospecimens from

377 participants consented at our satellite clinic, the Cornell University Veterinary Specialist in

378 Stamford, Connecticut, were also included.

380 Out of the 1,122 cats, 57 were recruited through the Senior Feline Health Screening program from

3812014 to 2018 . The program was created to build a biobank of DNA and associated clinical data

382 from healthy senior cats to serve as universal controls for mapping studies. In order to participate,

383 feline candidates had to be at least 9.5 years of age and in good health. Privately owned cats that

384 participated in the screening had a general physical examination and were examined accordingly

385 by board certified specialists: cardiac auscultation and echocardiogram, dental examination, body 
condition scoring, body mapping (used by oncologists to record any masses found), ocular examination, and an orthopedic examination. A complete blood count, serum chemistry panel, coagulation panel, feline immunodeficiency virus (FIV) and feline leukemia virus (FeLV) test,

389 baseline serum thyroxine (T4) level, and urinalysis were performed.

\section{Sample Processing, Storage and Distribution}

Samples were collected according to the Cornell University Institutional Animal Care and

393 Use Committee (IACUC) protocol \#2005-0151. Following owner informed consent, whole blood

394 samples were collected in EDTA tubes and refrigerated at $4^{\circ} \mathrm{C}$ until DNA extraction. Formalin

395 fixed, paraffin embedded (FFPE) scrolls of splenic tissue were acquired from a collaborating

396 pathologist and used for DNA extraction when necessary. Genomic DNA was extracted from

397 blood samples using a standard salt precipitation. Genomic DNA was extracted from FFPE 398 samples using the E.Z.N.A. Tissue DNA kit (Omega Bio-Tek) following the manufacturer's 399 instructions. DNA concentration and purity were determined by spectrophotometry on a 400 NanoDrop ND1000 (Thermo Scientific). DNA samples were stored at $\leq-20^{\circ} \mathrm{C}$ until distribution 401 for genotyping.

402

\section{Inclusion criteria}

Participants with a disease of interest could simultaneously be used as controls for other

405 traits/diseases, as long as these traits were ruled out. Phenotypes included cases and controls from 406 any breed, unless specified. Controls were at least 9.5 years of age, while cases could be of any 407 age. Numbers of purebred and random bred cats included as cases and controls for each GWAS 
408 are shown in Table 2 and numbers of individuals from each breed are shown in S5 Table. The

409 distribution of cases and controls by age is shown for each phenotype in S4 Fig.

410

411 Table 2: Distribution of cases and controls that are purebred and random bred cats for each

\section{2 disease.}

\begin{tabular}{|c|c|c|c|}
\hline Disease & $\begin{array}{c}\text { PUREBRED } \\
\text { Number cases, number } \\
\text { controls }\end{array}$ & $\begin{array}{l}\text { RANDOM BRED^ } \\
\text { Number cases, } \\
\text { number controls }\end{array}$ & $\begin{array}{l}\text { Total number } \\
\text { cases, controls }\end{array}$ \\
\hline HCM & 14,20 & 69,33 & 83,53 \\
\hline Hyperthyroidism & 24,38 & 286,96 & 310,134 \\
\hline DM & 13,62 & 54,304 & 67,366 \\
\hline CKD & 29,10 & 125,52 & 154,62 \\
\hline Chronic enteropathy & 9,10 & 32,18 & 41,28 \\
\hline IBD & 9,15 & 38,18 & 47,33 \\
\hline SCAL & 12,16 & 66,18 & 78,34 \\
\hline GI combined & 33,16 & 133,18 & 166,34 \\
\hline FEK & 2,13 & 13,27 & 15,40 \\
\hline Hypercalcemia & 5,78 & 20,365 & 25,443 \\
\hline
\end{tabular}


416 Hypertrophic cardiomyopathy. HCM is the most common cardiac disease in cats, affecting

417 around $15 \%$ of the feline population [51,52]. Similarly to humans, familial HCM has been

418 described in purebred cats, and in Maine coon and Ragdolls is caused by mutations in myosin

419 binding protein C gene (MYBPC3) [53]. Some Maine coon and Ragdolls cats develop HCM in

420 the absence of this mutation, indicating that other mutations are yet to be identified [53]. Diagnosis

421 was based on echocardiography. Phenotypic criteria for controls included normal left ventricular

422 wall thickness measurements: left ventricular free wall (LVFW) and interventricular septum (IVS)

423 in diastole $\leq 6 \mathrm{~mm}$ by M-mode (motion mode). Phenotypic criteria for cases included LVFW and

424 IVS wall thickness $>6 \mathrm{~mm}$. Additionally, affected cats must have had normal baseline T4 and be

425 normotensive and normally hydrated in order to rule out other causes of cardiac hypertrophy.

427 Hyperthyroidism. Hyperthyroidism is one of the most common endocrine disorders affecting

428 senior cats. The disease most often results from benign adenomatous thyroid nodules similar to

429 human toxic nodular goiter [54]. Hyperthyroidism is believed to be a multifactorial disease, with

430 nutritional, environmental, and genetic factors postulated as interacting causes [54]. The diagnosis

431 of cases and controls was based on the following criteria: control cats had low-normal thyroxine

432 (T4; $<3 \square \mathrm{g} / \mathrm{dL}$; normal range 2-5 $\square \mathrm{g} / \mathrm{dL}$ ). Cases had T4 $>5 \square \mathrm{g} / \mathrm{dL}$ or normal T4 with increased

433 free T4. Radioiodinated thyroid scan results confirming the diagnosis were recorded, if available.

435 Diabetes mellitus. DM is also one of the most common endocrine diseases of cats with the

436 majority of the cats resembling Type 2 (adult onset) DM in humans. The disease is caused by a 437 combination of decreased $\beta$-cell function, insulin resistance, and environmental and genetic factors 438 [55]. Diagnosis of DM was based on the following criteria: control cats had blood glucose values 
$439<200 \mathrm{mg} / \mathrm{dL}$ (reference values: 71-182 mg/dL) and no glucosuria. Cases had elevated blood

440 glucose $(>250 \mathrm{mg} / \mathrm{dL})$ and glucosuria in at least two consecutive visits. Also, fructosamine, if

441 evaluated, had to be above normal range (174-294 $\mu \mathrm{mol} / \mathrm{L})$. Of the 67 cases in the GWAS, 39 had

442 fructosamine tests and all had elevated levels. Fifty-three diabetic cases and 339 controls had body

443 weight recorded. Although the body weights of cases were spread throughout the range of $1.8 \mathrm{~kg}$

444 to $10 \mathrm{~kg}$, a greater proportion of cases (12 of 53 , or $22.6 \%)$ had weights $>7 \mathrm{~kg}$, compared to controls

445 (17 of 339, or 5.0\%) (S5 Fig.).

446

447 Chronic kidney disease. $\mathrm{CKD}$ is highly prevalent in both humans and cats with

448 approximately $10 \%$ of cats $>10$ years of age reported to be affected. Cats with CKD experience a

449 progressive loss of functional renal mass. CKD is considered a heterogeneous syndrome, rather

450 than a single entity [56]. CKD was diagnosed by evaluating the level of blood urea nitrogen (BUN)

451 and creatinine, in conjunction with the urine specific gravity (USG). Symmetric dimethylarginine

452 (SDMA), a natural occurring indicator for kidney function, was measured in the blood of some

453 cases to determine if early renal disease was occurring. The diagnosis was established according

454 to the following criteria: controls cats had creatinine $<1.6 \mathrm{mg} / \mathrm{dL}$ (normal range $0.6-2 \mathrm{mg} / \mathrm{dL}$ ),

455 BUN within normal range (16-36 mg/dL) and USG $>1.035$ (preferably performed on the same day

456 as creatinine was measured). Cases had to be azotemic (elevated BUN and creatinine values) with

457 concurrent isosthenuria (failure of the kidney to dilute or concentrate urine) and increased SDMA,

458 diagnosed by a board-certified veterinary internist.

460 Chronic enteropathy/inflammatory bowel disease/small cell alimentary

461 lymphoma. Chronic enteropathies, which include Inflammatory Bowel Disease (IBD) and 
462 Small Cell Alimentary Lymphoma (SCAL), are common forms of primary gastrointestinal disease

463 in cats. Although the cause of feline IBD is unknown, it has been hypothesized that, similar to

464 canines and humans, feline IBD is caused by several factors such as intestinal microbial

465 imbalances, diet, and defects in the mucosal immune system [57]. SCAL is the most frequent

466 digestive neoplasia in cats, accounting for 60-75\% of gastrointestinal lymphoma cases [58].

468 For this study, cats were assigned as chronic enteropathy cases if gastrointestinal (GI) clinical signs

469 such as chronic vomiting, diarrhea, or weight loss were present, non-GI causes of their clinical

470 signs were excluded, thus highly suggestive of either IBD or SCAL, but no histologic diagnosis

471 was performed. IBD and SCAL were considered separate diagnoses that required histological

472 confirmation. Distinguishing between IBD and SCAL can be difficult, so in addition to histologic

473 assessment, immunophenotyping and polymerase chain reaction (PCR) for antigen receptor

474 rearrangements (PARR) were used in some cases to confirm the SCAL diagnosis. Phenotypic

475 criteria for affected cats included persistent clinical GI signs and histopathology performed by a

476 board-certified veterinary pathologist confirming either IBD or SCAL. Control cats were

477 examined by a board-certified oncologist and had an absence of any GI signs. We performed a

478 separate GWAS for each of IBD and SCAL, and then chronic enteropathy, which includes cats

479 that were not formally diagnosed but could be either IBD or SCAL. Finally, we performed a

480 GWAS including all GI cases in an attempt to increase statistical power, and since IBD, SCAL,

481 and chronic enteropathy can be considered a different manifestation of the same disorder [59].

482 There is also evidence that IBD leads to SCAL [60]. 
484 Feline eosinophilic keratoconjunctivitis. FEK is a corneal/conjunctival disease

485 characterized by vascularized white-to-pink plaques on the cornea and bulbar conjunctiva. In the

486 majority of cats, previous corneal ulceration has been diagnosed and an association with feline

487 herpesvirus type $1(\mathrm{FHV}-1)$ infection has been proposed [61]. The diagnosis of FEK was made 488 according to the following criteria: affected cats had signs of the disease during ophthalmologic

489 exam performed by a board-certified veterinary ophthalmologist, including proliferative

490 vascularized lesions affecting peripheral corneal/bulbar conjunctiva and the presence of 491 eosinophils in the ocular cytology. Control cats had a normal ophthalmologic exam.

492

493 Hypercalcemia. Hypercalcemia is a common condition of cats defined by an increase in both

494 total and ionized serum calcium. It may be caused by many conditions such as neoplasia, renal

495 failure, primary hyperparathyroidism, hypoadrenocorticism, ingestion of cholecalciferol-

496 containing rodenticides, or granulomatous disease. In cats, hypercalcemia can also be idiopathic

497 [62], which is the phenotype we are investigating here. The diagnosis of hypercalcemia was

498 determined as follows: control cats had total serum calcium values within the normal range (9.1-

$49910.9 \mathrm{mg} / \mathrm{dL}$ ); affected cats had elevated total serum calcium and ionized calcium values (reference

500 interval 1.11-1.38 mmol/L). Parathyroid hormone $(\mathrm{PTH})$ and PTH related peptide $(\mathrm{PTHrP})$ were

501 recorded if available, and were used to differentiate between causes of hypercalcemia.

\section{Design of array}

504 Genotyping was performed on an Illumina Infinium iSelect Custom BeadChip. These 505 arrays contain 340,000 attempted beadtypes for genotyping single nucleotide polymorphisms 
506 selected across the entire cat genome, using feline genome assembly felCat5. Of the 340,000

507 markers included on the array, 297,034 (87\%) provided a reliable call.

508

509 SNPs for the array were selected from whole genome sequencing of 6 genetically diverse female

510 DSH cats. These 6 cats were sequenced on a HiSeq2500 (Illumina, San Diego, CA) to generate

511 100bp paired-end reads. Following GATK best practices pipeline [63], reads were mapped to the

512 feline reference genome using BWA mem [64], then duplicate reads were tagged by PICARD

513 MarkDuplicates, and indels were realigned and quality scores were recalibrated using GATK.

514 Variants were called and filtered using GATK HaplotypeCaller and VCFtools [65]. The full list of

515 variants was thinned randomly using PLINK and then protein-coding variants with moderate and

516 high impact as defined by SnpEff [66] were added back in.

\section{Genotyping}

In total, 1,200 feline DNA samples were genotyped on the Hill's custom Illumina feline

520 high density mapping array. Genotyping was performed in 11 batches, or plates, by Neogen

521 GeneSeek Operations (Lincoln, NE). Raw data files were converted to PLINK format and quality

522 control was performed in PLINK v1.9 (www.cog-genomics.org/plink/1.0/) $[67,68]$.

\section{Quality control}

Genotyping data from the 11 batches were merged together using PLINK's --bmerge

526 command and a sex check of all samples was performed using PLINK's --check-sex command.

527 Seventy samples were removed due to missingness $>80 \%$, including 53 samples from the same

528 batch. 
530 SNPs were converted to the genome assembly felCat9 [69] and SNPs with missingness $>95 \%$ in

531 the 1,130 cats were removed, leaving 252,987 SNPs. Eight cats were genotyped on two different

532 plates each as internal controls. The SNPs that were discordant between these eight duplicates

533 were identified and removed. Finally, duplicate samples were removed, leaving a dataset of 1,122

534 individuals and 251,978 SNPs for GWAS.

535

536 A Principal Component Analysis (PCA) was performed using the program EIGENSTRAT in the 537 EIGENSOFT package [70]. For this, linked SNPs were pruned using PLINK's --indep 5052 538 command, leaving 91,556 SNPs. PCA was performed using all cats to look for batch effects, and

539 all purebred cats to ensure individuals of the same breed clustered together. PCA was also 540 performed using only the cats included in each phenotype to identify and remove outliers before 541 GWAS analysis. An outlier is an individual that is located separately from the main cluster of cats 542 on either the PC1 or PC2 axis. Further, in order to reduce the effects of genetically distinct 543 individuals in our GWAS, we also removed any purebred cat that was located separately from the 544 main cluster of random bred individuals on either PC1 or PC2.

546 For the DM and HCM phenotypes, a further two and 16 cats, respectively, were genotyped on the 547 same 340k custom Illumina array by external coauthors (MEW and JAB, respectively). For these 548 cats, the genotype files were merged with the sample set before the QC was performed, as 549 described above. The genotype and phenotype data for all three of these datasets are available as 550 PLINK files, and include the SNP information (chromosome, bp location, alleles). 


\section{Genome-wide association study}

Both a single-locus linear mixed model (LMM) and a multi-locus model were used to

554 perform a GWAS for each disease phenotype. The LMM was performed in the program GEMMA

555 v 0.98.1 [71], which includes a relatedness matrix as a random effect. The multi-locus method

556 performed was FarmCPU (Fixed and random model Circulating Probability Unification) [72] run

557 using rMVP [73] in R. FarmCPU is designed to help control for false positives by including

558 associated markers as covariates, while also reducing false negatives by removing the confounding

559 between the population structure and kinship and the markers to be tested. We used the default

560 parameters, with a maximum of 10 iterations. For each phenotype, we included the relatedness

561 matrix calculated by GEMMA and a covariate file consisting of the first four PCs from a PCA run

562 on the genotypes of the cats included in the phenotype only.

564 For both models, the Wald test was used to calculate $P$-values, and the Bonferroni correction

$565\left(\mathrm{p}_{\text {genome }}=0.05\right)$ was used to calculate the genome-wide significance threshold. A suggestive

566 threshold was calculated using the Bonferroni correction on unlinked SNPs (pruned using the --

567 indep 5052 option in PLINK).

569 For each phenotype, PCA outliers and related cats (pihat $>0.40$ ) were excluded. Single nucleotide

570 polymorphisms with a minor allele frequency (MAF) $<5 \%$ and a genotyping call rate $<90 \%$ were

571 removed from each analysis. SNPs are provided in genome assembly felCat9. 
573 Manhattan and quantile-quantile (QQ) plots were created using the package qqman [74] in R

574 v4.0.2 [75]. Lambda values, as a quantification for genomic inflation, were calculated in R.

575 Linkage disequilibrium plots were created using matplolib [76] in jupyter notebook [77].

\section{GWAS positive controls}

As a positive control for the 340k array, we performed GWAS on the presence of orange

fur. The Orange locus has been refined to a $1.5 \mathrm{Mb}$ region on the $\mathrm{X}$ chromosome, although the causal variant is unknown [16-18]. We used 211 random bred cats in the orange GWAS: 90 cats

581 that had a coat color description of orange (including solid orange, orange and white, and orange

582 tabby), and 121 cats that had a coat color description of black, brown or brown tabby.

584 We also performed a positive control GWAS of factor XII deficiency, a common hereditary coagulation factor deficiency in cats that does not cause a bleeding diathesis. For this phenotype,

586 affected cats were classified based on severe factor XII deficiency (factor XII coagulant activity $<$

$58710 \%$ of normal), whereas control cats had values above $60 \%$. Nineteen affected cats and 34 588 controls were included in the GWAS.

590 Acknowledgements

We especially thank the faculty and staff of the Cornell University Hospital for Animals

592 for sample collection and phenotyping of control cats. We would like to acknowledge Gregory

593 Acland and John Schimenti for instigation of the Cornell Veterinary Biobank, and support from

594 Baker Institute for Animal Health, Cornell University Center for Vertebrate Genomics, and 
Department of Clinical Sciences. Finally, we thank numerous pet owners for donating their pets' samples, and collaborators for sample collection and phenotyping.

\section{References}

599 1. AVMA Pet Ownership and Demographics Sourcebook. 2017-2018 edition. Schaumburg,IL:

$600 \quad$ American Veterinary Medical Association, 2018.

601 2. Driscoll CA, Clutton-Brock J, Kitchener AC, O’Brien SJ. The Taming of the cat. Genetic 602 and archaeological findings hint that wildcats became housecats earlier--and in a different 603 place--than previously thought. Sci Am. 2009;300: 68-75.

604 3. Alhaddad H, Khan R, Grahn RA, Gandolfi B, Mullikin JC, Cole SA, et al. Extent of 605 Linkage Disequilibrium in the Domestic Cat, Felis silvestris catus, and Its Breeds. PLoS ONE. 2013;8. doi:10.1371/journal.pone.0053537

4. Takaichi Y, Chambers JK, Shiroma-Kohyama M, Haritani M, Une Y, Yamato O, et al. Feline Spongy Encephalopathy With a Mutation in the ASPA Gene. Vet Pathol. 2021; 3009858211002176. doi:10.1177/03009858211002176

5. Li RHL, Ontiveros E, Nguyen N, Stern JA, Lee E, Hardy BT, et al. Precision medicine identifies a pathogenic variant of the ITGA2B gene responsible for Glanzmann's thrombasthenia in a cat. J Vet Intern Med. 2020;34: 2438-2446. doi:10.1111/jvim.15886 variant in the NSDHL gene in a cat with multiple congenital lesions resembling 
615 inflammatory linear verrucous epidermal nevi. Vet Dermatol. 2019;30: 64-e18.

616 doi:10.1111/vde.12699

617 7. Baker L, Muir P, Sample SJ. Genome-wide association studies and genetic testing:

618 Understanding the science, success, and future of a rapidly developing field. Journal of the

619 American Veterinary Medical Association. American Veterinary Medical Association;

620 2019. pp. 1126-1136. doi:10.2460/javma.255.10.1126

621 8. Gandolfi B, Gruffydd-Jones TJ, Malik R, Cortes A, Jones BR, Helps CR, et al. First WNK4-Hypokalemia Animal Model Identified by Genome-Wide Association in Burmese Cats. PLoS ONE. 2012;7. doi:10.1371/journal.pone.0053173

9. Alhaddad H, Gandolfi B, Grahn RA, Rah HC, Peterson CB, Maggs DJ, et al. Genome-wide association and linkage analyses localize a progressive retinal atrophy locus in Persian cats. Mamm Genome. 2014;25: 354-362. doi:10.1007/s00335-014-9517-z

10. Gandolfi B, Grahn RA, Creighton EK, Williams DC, Dickinson PJ, Sturges BK, et al. COLQ variant associated with Devon Rex and Sphynx feline hereditary myopathy. Anim Genet. 2015;46: 711-715. doi:10.1111/age.12350 Like Homeobox protein 1 (ALX1) variant associated with craniofacial structure and

632 frontonasal dysplasia in Burmese cats. Dev Biol. 2016;409: 451-458.

$633 \quad$ doi:10.1016/j.ydbio.2015.11.015 
634 12. Yu Y, Creighton EK, Buckley RM, Lyons LA. A deletion in GDF7 is associated with a heritable forebrain commissural malformation concurrent with ventriculomegaly and interhemispheric cysts in cats. Genes. 2020;11: 1-15. doi:10.3390/genes11060672

13. Gandolfi B, Alamri S, Darby WG, Adhikari B, Lattimer JC, Malik R, et al. A dominant TRPV4 variant underlies osteochondrodysplasia in Scottish fold cats. Osteoarthritis Cartilage. 2016;24: 1441-1450. doi:10.1016/j.joca.2016.03.019

14. Golovko L, Lyons LA, Liu H, Sørensen A, Wehnert S, Pedersen NC. Genetic susceptibility to feline infectious peritonitis in Birman cats. Virus Res. 2013;175: 58-63.

643 15. Samaha G, Wade CM, Beatty J, Lyons LA, Fleeman LM, Haase B. Mapping the genetic doi:10.1016/j.virusres.2013.04.006

16. Gandolfi B, Alhaddad H, Abdi M, Bach LH, Creighton EK, Davis BW, et al. Applications and efficiencies of the first cat 63K DNA array. Sci Rep. 2018;8. doi:10.1038/s41598-018domestic cat X chromosome linkage map and the sex-linked orange locus: Mapping of orange, multiple origins and epistasis over nonagouti. Genetics. 2009;181: 1415-1425. doi:10.1534/genetics.108.095240 
653 18. Grahn RA, Lemesch BM, Millon L V., Matise T, Rogers QR, Morris JG, et al. Localizing

654 the X-linked orange colour phenotype using feline resource families. Anim Genet. 2005;36: 67-70. doi:10.1111/j.1365-2052.2005.01239.x

19. Maruyama H, Brooks MB, Stablein A, Frye A. Factor XII deficiency is common in domestic cats and associated with two high frequency F12 mutations. Gene. 2019;706: 612. doi:10.1016/j.gene.2019.04.053

20. O’Neill DG, Church DB, McGreevy PD, Thomson PC, Brodbelt DC. Prevalence of disorders recorded in cats attending primary-care veterinary practices in England. Vet J.

21. Kurushima JD, Lipinski MJ, Gandolfi B, Froenicke L, Grahn JC, Grahn RA, et al. Variation of cats under domestication: Genetic assignment of domestic cats to breeds and worldwide ascent of cat breeds: Genetic evaluations of breeds and worldwide random-bred populations. Genomics. 2008;91: 12-21. doi:10.1016/j.ygeno.2007.10.009 cat breeds assessed by a 63K SNP array. Barsh GS, editor. PLOS ONE. 2021;16: e0247092. doi:10.1371/journal.pone.0247092 
673 25. Desikan RS, Schork AJ, Wang Y, Thompson WK, Dehghan A, Ridker PM, et al. Polygenic 674 Overlap Between C-Reactive Protein, Plasma Lipids, and Alzheimer Disease. Circulation. 2015;131: 2061-2069. doi:10.1161/CIRCULATIONAHA.115.015489

676

677

678

26. Sun K, He S-B, Yao Y-Z, Qu J-G, Xie R, Ma Y-Q, et al. Tre2 (USP6NL) promotes colorectal cancer cell proliferation via Wnt/ $\beta$-catenin pathway. Cancer Cell Int. 2019;19: 102. doi:10.1186/s12935-019-0823-0

27. Avanzato D, Pupo E, Ducano N, Isella C, Bertalot G, Luise C, et al. High USP6NL Levels in Breast Cancer Sustain Chronic AKT Phosphorylation and GLUT1 Stability Fueling Aerobic Glycolysis. Cancer Res. 2018;78: 3432-3444. doi:10.1158/0008-5472.CAN-173018

28. Yamagata M, Weiner JA, Sanes JR. Sidekicks: synaptic adhesion molecules that promote lamina-specific connectivity in the retina. Cell. 2002;110: 649-660. doi:10.1016/s00928674(02)00910-8

29. Kaufman L, Potla U, Coleman S, Dikiy S, Hata Y, Kurihara H, et al. Up-regulation of the homophilic adhesion molecule sidekick-1 in podocytes contributes to glomerulosclerosis. J Biol Chem. 2010;285: 25677-25685. doi:10.1074/jbc.M110.133959

30. Oguri M, Kato K, Yokoi K, Yoshida T, Watanabe S, Metoki N, et al. Assessment of a Polymorphism of SDK1 With Hypertension in Japanese Individuals. Am J Hypertens. 2010;23: 70-77. doi:10.1038/ajh.2009.190 
692 31. Tjoelker LW, Stafforini DM. Platelet-activating factor acetylhydrolases in health and

693 disease. Biochimica et Biophysica Acta - Molecular and Cell Biology of Lipids. Biochim

694 Biophys Acta; 2000. pp. 102-123. doi:10.1016/S1388-1981(00)00114-1

695 32. Rubin CI, Atweh GF. The role of stathmin in the regulation of the cell cycle. J Cell

696 Biochem. 2004;93: 242-250. doi:10.1002/jcb.20187

697 33. Hayward JJ, Castelhano MG, Oliveira KC, Corey E, Balkman C, Baxter TL, et al. Complex

698 disease and phenotype mapping in the domestic dog. Nat Commun. 2016;7.

699 doi: $10.1038 /$ ncomms 10460

700 34. Kent WJ. BLAT---The BLAST-Like Alignment Tool. Genome Res. 2002;12: 656-664.

701 doi: $10.1101 / \mathrm{gr} .229202$

702 35. Soria JM, Almasy L, Souto JC, Bacq D, Buil A, Faure A, et al. A Quantitative-Trait Locus

703 in the Human Factor XII Gene Influences Both Plasma Factor XII Levels and Susceptibility

704 to Thrombotic Disease. Am J Hum Genet. 2002;70: 567-574.

705 36. You J, Chen W, Chen J, Zheng Q, Dong J, Zhu Y. The oncogenic role of ARG1 in

706 progression and metastasis of hepatocellular carcinoma. BioMed Res Int. 2018;2018.

707 doi: $10.1155 / 2018 / 2109865$

708 37. Olateju TO, Vanderpump MPJ. Thyroid hormone resistance. Annals of Clinical

709 Biochemistry. Ann Clin Biochem; 2006. pp. 431-440. doi:10.1258/000456306778904678 
710 38. Watson SG, Radford AD, Kipar A, Ibarrola P, Blackwood L. Somatic mutations of the thyroid-stimulating hormone receptor gene in feline hyperthyroidism: Parallels with human

39. Precone V, Beccari T, Stuppia L, Baglivo M, Paolacci S, Manara E, et al. Taste, olfactory and texture related genes and food choices: Implications on health status. Eur Rev Med Pharmacol Sci. 2019;23: 1305-1321.doi:10.26355/eurrev_201902_17026

40. Ramos-Lopez O, Riezu-Boj JI, Milagro FI, Zulet MA, Santos JL, Martinez JA, et al. Associations between olfactory pathway gene methylation marks, obesity features and dietary intakes. Genes Nutr. 2019;14. doi:10.1186/s12263-019-0635-9

41. Munakata Y, Yamada T, Imai J, Takahashi K, Tsukita S, Shirai Y, et al. Olfactory receptors are expressed in pancreatic $\beta$-cells and promote glucose-stimulated insulin secretion. Sci Rep. 2018;8. doi:10.1038/s41598-018-19765-5

42. Robertson RP. Prostaglandins, glucose homeostasis, and diabetes mellitus. Annual review of medicine. Annu Rev Med; 1983. pp. 1-12. doi:10.1146/annurev.me.34.020183.000245

43. Attia HRM, Kamel SA, Ibrahim MH, Farouk HA, Rahman AHA, Sayed GH, et al. Openarray analysis of genetic variants in Egyptian patients with type 2 diabetes and obesity. Egypt J Med Hum Genet. 2017;18: 341-348. doi:10.1016/j.ejmhg.2017.03.002

727 44. Tang H, Wei P, Duell EJ, Risch HA, Olson SH, Bueno-De-Mesquita HB, et al. Genes728 environment interactions in obesity- and diabetes-associated pancreatic cancer: A GWAS 729 data analysis. Cancer Epidemiol Biomarkers Prev. 2014;23: 98-106. doi:10.1158/10559965.EPI-13-0437-T 
731 45. Balmer L, O’leary CA, Menotti-Raymond M, David V, O’brien S, Penglis B, et al.

732 Mapping of diabetes susceptibility LOCI in a domestic cat breed with an unusually high incidence of diabetes mellitus. Genes. 2020;11: 1-11. doi:10.3390/genes 11111369

46. Forcada Y, Holder A, Church DB, Catchpole B. A Polymorphism in the melanocortin 4 receptor gene (MC4R: C.92C $>$ T) is associated with diabetes mellitus in overweight domestic shorthaired cats. J Vet Intern Med. 2014;28: 458-464. doi:10.1111/jvim.12275

47. Holgate ST. Epithelial damage and response. Clinical and Experimental Allergy, Supplement. Clin Exp Allergy; 2000. pp. 37-41. doi:10.1046/j.1365-2222.2000.00095.x

48. Yang L, Cohn L, Zhang DH, Homer R, Ray A, Ray P. Essential role of nuclear factor $\kappa \mathrm{B}$ in the induction of eosinophilia in allergic airway inflammation. J Exp Med. 1998;188: 17391750. doi:10.1084/jem.188.9.1739

49. Collins PD, Marleau S, Griffiths-Johnson DA, Jose PJ, Williams TJ. Cooperation between interleukin-5 and the chemokine eotaxin to induce eosinophil accumulation in vivo. J Exp expression and TH2 differentiation in allergic airway inflammation. Nat Immunol. 2001;2: Med. 1995;182: 1169-1174. doi:10.1084/jem.182.4.1169 45-50. doi:10.1038/83158 healthy cats in rehoming centres (the CatScan study). J Vet Cardiol. 2015;17: S244-S257. doi:10.1016/j.jvc.2015.03.008 
52. Gil-Ortuño C, Sebastián-Marcos P, Sabater-Molina M, Nicolas-Rocamora E, Gimeno-

53. Côté E, MacDonald KA, Meurs KM, Sleeper MM. Hypertrophic Cardiomyopathy. In:

54. Peterson ME. Feline hyperthyroidism: An animal model for toxic nodular goiter. Journal of Endocrinology. BioScientifica Ltd.; 2014. pp. T97-T114. doi:10.1530/JOE-14-0461

55. O’Neill DG, Gostelow R, Orme C, Church DB, Niessen SJM, Verheyen K, et al. Epidemiology of Diabetes Mellitus among 193,435 Cats Attending Primary-Care Veterinary Practices in England. J Vet Intern Med. 2016;30: 964-972. doi:10.1111/jvim. 14365

56. Finch NC, Syme HM, Elliott J. Risk Factors for Development of Chronic Kidney Disease in Cats. J Vet Intern Med. 2016;30: 602-610. doi:10.1111/jvim.13917

57. Jergens AE. Feline Idiopathic Inflammatory Bowel Disease: What we know and what remains to be unraveled. J Feline Med Surg. 2012;14: 445-458. doi:10.1177/1098612X12451548 low-grade alimentary lymphoma: An emerging entity and a potential animal model for human disease. BMC Veterinary Research. BioMed Central Ltd.; 2018. doi:10.1186/s12917-018-1635-5 
59. Marsilio S. Feline chronic enteropathy. J Small Anim Pract. 2021;62: 409-419. doi:10.1111/jsap.13332

60. Moore PF, Woo JC, Vernau W, Kosten S, Graham PS. Characterization of feline T cell receptor gamma (TCRG) variable region genes for the molecular diagnosis of feline intestinal T cell lymphoma. Vet Immunol Immunopathol. 2005;106: 167-178. doi:10.1016/j.vetimm.2005.02.014

61. Lucyshyn DR, Good KL, Knickelbein KE, Chang MW, Strøm AR, Hollingsworth SR, et al. Subcutaneous administration of triamcinolone as part of the management of feline eosinophilic keratoconjunctivitis. J Feline Med Surg. 2020. doi:10.1177/1098612X20968660

62. Midkiff AM, Chew DJ, Randolph JF, Center SA, DiBartola SP. Idiopathic Hypercalcemia in Cats. J Vet Intern Med. 2000;14: 619. doi:10.1892/08916640(2000)014<0619:ihic >2.3.co;2

63. Van der Auwera GA, Carneiro MO, Hartl C, Poplin R, del Angel G, Levy-Moonshine A, et al. From fastQ data to high-confidence variant calls: The genome analysis toolkit best practices pipeline. Curr Protoc Bioinforma. 2013;43. doi:10.1002/0471250953.bi1110s43

64. Li H, Durbin R. Fast and accurate short read alignment with Burrows-Wheeler transform. Bioinformatics. 2009;25: 1754-1760. doi:10.1093/bioinformatics/btp324

65. Danecek P, Auton A, Abecasis G, Albers CA, Banks E, DePristo MA, et al. The variant call format and VCFtools. Bioinformatics. 2011;27: 2156-2158. doi:10.1093/bioinformatics/btr330 
792 66. Cingolani P, Platts A, Wang LL, Coon M, Nguyen T, Wang L, et al. A program for

793

794

795

796

797

798

799

800

801

802

803

804

805

806

807

808

809

810

annotating and predicting the effects of single nucleotide polymorphisms, SnpEff: SNPs in the genome of Drosophila melanogaster strain w1118; iso-2; iso-3. Fly (Austin). 2012;6: 80-92. doi:10.4161/fly.19695

67. Chang CC, Chow CC, Tellier LCAM, Vattikuti S, Purcell SM, Lee JJ. Second-generation PLINK: Rising to the challenge of larger and richer datasets. GigaScience. 2015;4. doi:10.1186/s13742-015-0047-8

68. Purcell S, Neale B, Todd-Brown K, Thomas L, Ferreira MAR, Bender D, et al. PLINK: A tool set for whole-genome association and population-based linkage analyses. Am J Hum Genet. 2007;81: 559-575. doi:10.1086/519795

69. Buckley RM, Davis BW, Brashear WA, Farias FHG, Kuroki K, Graves T, et al. A new domestic cat genome assembly based on long sequence reads empowers feline genomic medicine and identifies a novel gene for dwarfism. PLoS Genet. 2020;16. doi:10.1371/journal.pgen.1008926

70. Price AL, Patterson NJ, Plenge RM, Weinblatt ME, Shadick NA, Reich D. Principal components analysis corrects for stratification in genome-wide association studies. Nat Genet. 2006;38: 904-909. doi:10.1038/ng1847

71. Zhou X, Stephens M. Genome-wide efficient mixed-model analysis for association studies. Nat Genet. 2012;44: 821-824. doi:10.1038/ng.2310 
811 72. Liu X, Huang M, Fan B, Buckler ES, Zhang Z. Iterative Usage of Fixed and Random Effect

812 Models for Powerful and Efficient Genome-Wide Association Studies. PLoS Genet.

813 2016;12: e1005767. doi:10.1371/journal.pgen.1005767

814 73. Yin L, Zhang H, Tang Z, Xu J, Yin D, Zhang Z, et al. rMVP: A Memory-efficient,

815 Visualization-enhanced, and Parallel-accelerated tool for Genome-Wide Association Study.

816 Genomics Proteomics Bioinformatics. 2021; S1672-0229(21)00050-4.

817 doi:10.1016/j.gpb.2020.10.007

818 74. D. Turner S. qqman: an R package for visualizing GWAS results using Q-Q and manhattan

819 plots. J Open Source Softw. 2018;3: 731. doi:10.21105/joss.00731

820 75. R Core Team. R: A language and environment for statistical computing. R Found Stat

$821 \quad$ Comput. 2020.

822 76. Hunter JD. Matplotlib: A 2D graphics environment. Comput Sci Eng. 2007;9: 90-95.

823 doi: $10.1109 /$ MCSE.2007.55

824 77. Pérez F, Granger BE. IPython: A system for interactive scientific computing. Comput Sci Eng. 2007;9: 21-29. doi:10.1109/MCSE.2007.53 
S1 Fig. LD plots for the GWAS positive controls, using the LMM GWAS results. (A) Orange locus GWAS, showing the significant association on chromosome X. (B) Factor XII deficiency GWAS, showing the A1 significant association in the vicinity of the gene F12. Colors indicate the amount of LD $\left(\mathrm{r}^{2}\right)$ with the most significant SNP, ranging from black $\left(\mathrm{r}^{2}<0.2\right)$ to red $\left(\mathrm{r}^{2}>0.8\right)$. Manhattan plot, the red dashed line is the Bonferroni-corrected significance threshold. Inflation

836 factor $(\boldsymbol{\lambda})$ is shown on the QQ plot. unlinked SNPs. Inflation factors $(\boldsymbol{\lambda})$ are shown on QQ plots.

S4 Fig. Age distribution of cases and controls. (A) HCM, (B) hyperthyroidism, (C) DM, (D)

844 CKD, (E) chronic enteropathy, (F) IBD, (G) SCAL, (H) all GI, (I) FEK, (J) hypercalcemia. Age

845 (in months) is shown on the $\mathrm{X}$ axis and number of cats is shown on the $\mathrm{Y}$ axis. Cases are the pink 846 bars and controls are the blue bars.

848 S5 Fig. Weight distribution of cases and controls for DM. Weight (in $\mathrm{kg}$ ) is shown on the $\mathrm{X}$ 849 axis and number of cats is shown on the $\mathrm{Y}$ axis. Cases are the pink bars and controls are the blue 
850 bars. Note that weight data was only available for 53 of the 67 cases, and 339 of the 366 controls

851 used in the GWAS.

852

853 S1 Table. Significant association LMM GWAS results on chromosome $X$ for the presence of

854 orange fur in random bred cats.

855

856 S2 Table. Three most significant associations for each disease from the FarmCPU GWAS

857 results.

858

859 S3 Table. Frequencies, odds ratios, and $\boldsymbol{P}$-values for SNPs associated with complex diseases

860 in different breeds and random bred cats.

861

862 S4 Table. Non-significant associations identified for complex diseases using a LMM GWAS

863 design.

864

865 S5 Table. Numbers of individual cats from each breed and random bred group for each 866 phenotype. 


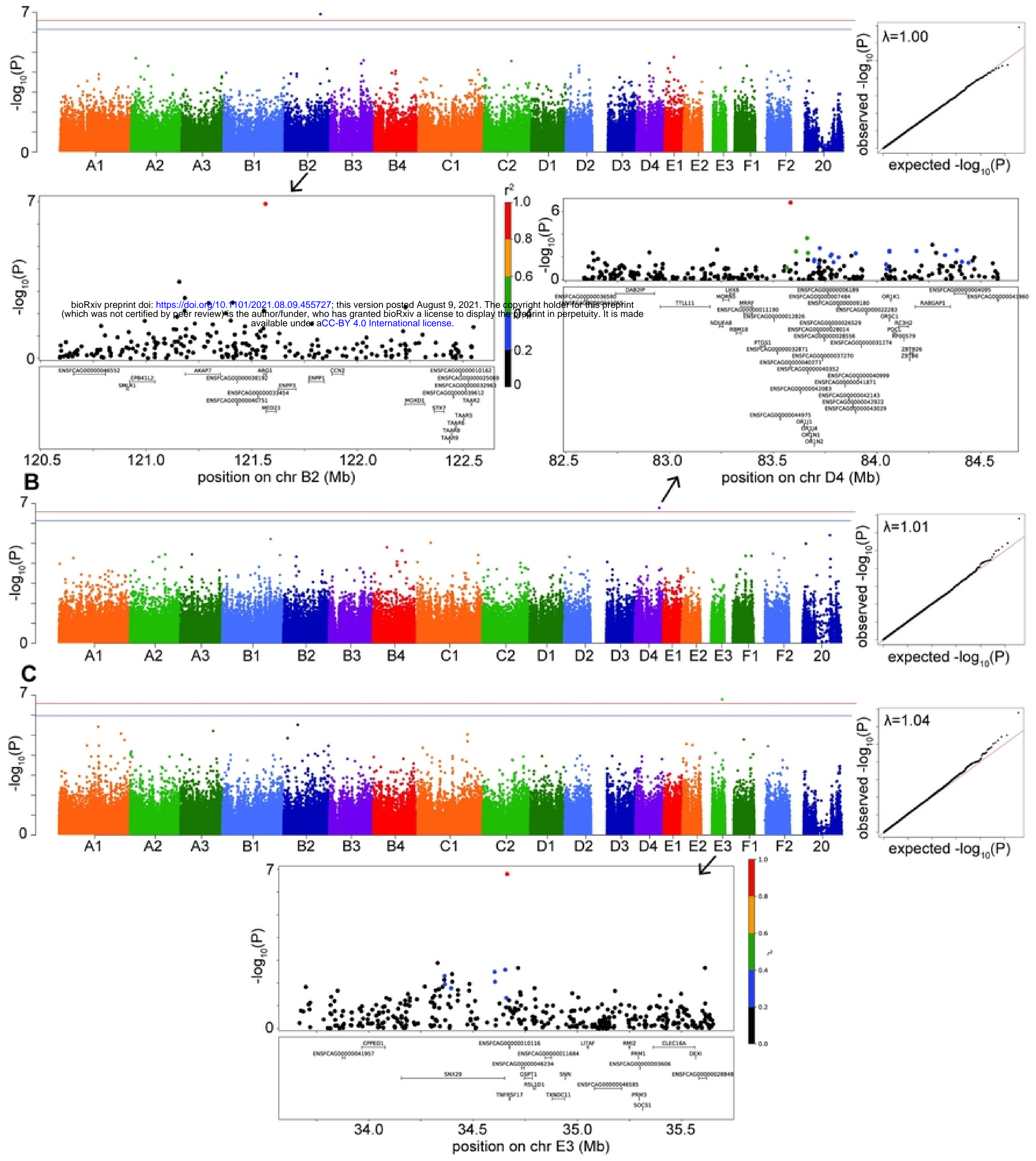

Figure 3 


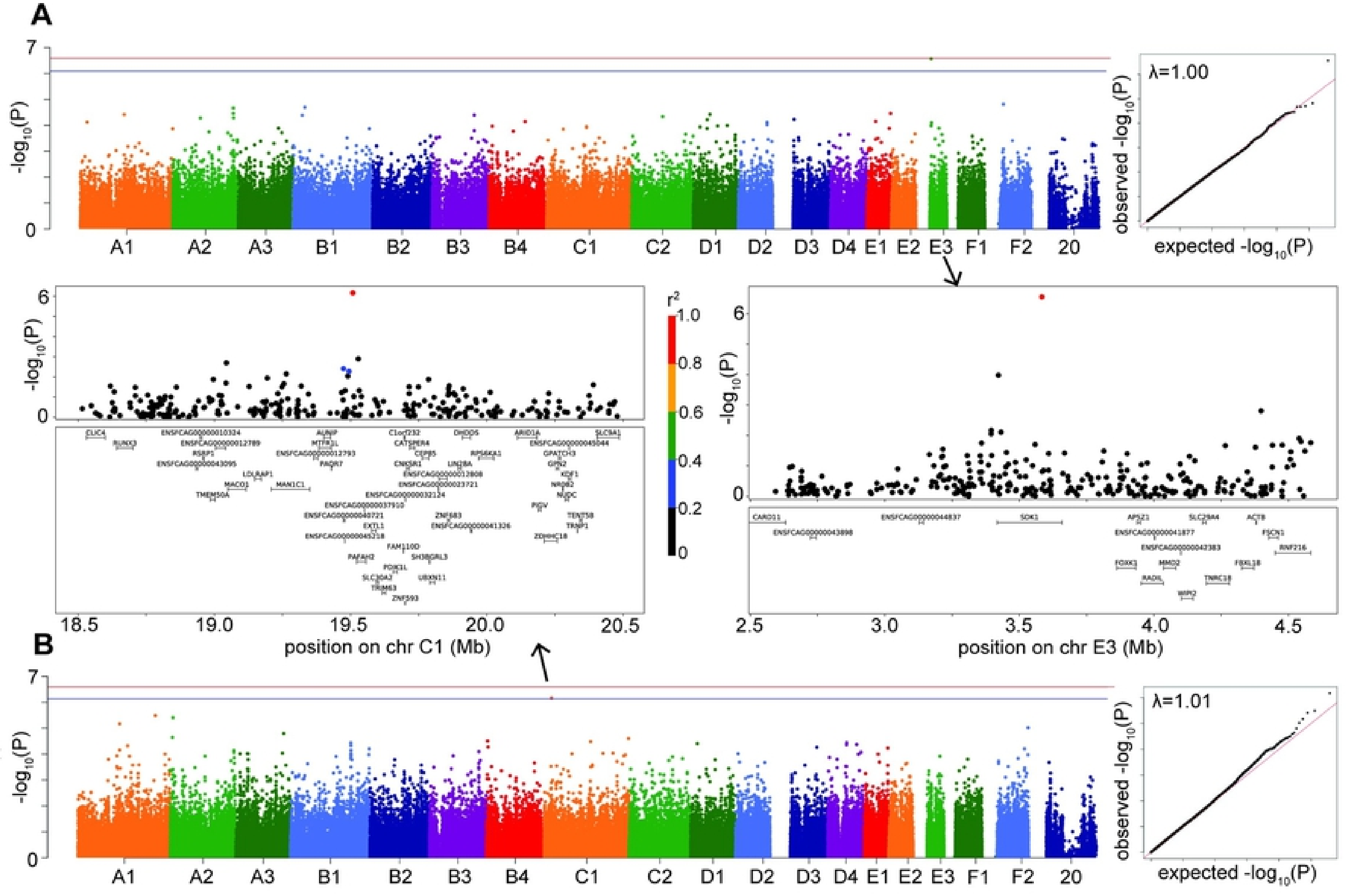

Figure 4 
